\title{
Review Article \\ Microfluidic Sample Preparation Methods for the Analysis of Milk Contaminants
}

\author{
Andrea Adami, Alessia Mortari, Elisa Morganti, and Leandro Lorenzelli
}

Centre for Materials and Microsystems, Fondazione Bruno Kessler, 38123 Trento, Italy

Correspondence should be addressed to Andrea Adami; andadami@fbk.eu

Received 14 August 2015; Accepted 5 November 2015

Academic Editor: Kourosh Kalantar-Zadeh

Copyright (C) 2016 Andrea Adami et al. This is an open access article distributed under the Creative Commons Attribution License, which permits unrestricted use, distribution, and reproduction in any medium, provided the original work is properly cited.

\begin{abstract}
In systems for food analysis, one of the major challenges is related to the quantification of specific species into the complex chemical and physical composition of foods, that is, the effect of "matrix"; the sample preparation is often the key to a successful application of biosensors to real measurements but little attention is traditionally paid to such aspects in sensor research. In this critical review, we discuss several microfluidic concepts that can play a significant role in sample preparation, highlighting the importance of sample preparation for efficient detection of food contamination. As a case study, we focus on the challenges related to the detection of aflatoxin $M_{1}$ in milk and we evaluate possible approaches based on inertial microfluidics, electrophoresis, and acoustic separation, compared with traditional laboratory and industrial methods for phase separation as a baseline of thrust and well-established techniques.
\end{abstract}

\section{Introduction}

In systems for food analysis, one of the major challenges is related to the quantification of specific species into the complex chemical and physical composition of foods, that is, the effect of "matrix"; in addition, target contaminants must be often detected at very low concentrations typically ranging from $\mathrm{ng} / \mathrm{kg}$ to $\mu \mathrm{g} / \mathrm{kg}$. The scenario is then quite different from other routine analysis, for example, fat and protein content in milk for quality evaluation of products during production, which is often based on high throughput IR spectroscopy methods or cytometry for somatic cells or bacteria quantification.

In the cases where the detection is based on surface interaction like in biosensors, food analysis is particularly sensitive to matrix interaction, ranging from unwanted surface coating or interaction by matrix components to segregation of target analytes into specific fractions of the sample, thus reducing the efficiency of capture by biorecognition molecules on the sensor. Therefore, it is common for sensors to have low sensitivity, specificity, reproducibility, and very short lifetime in food matrixes and it is often required to process the sample before quantification of the chemical species under examination in order to increase the analytical accuracy of a method. In the field of biosensors, the sample preparation often relies on time-consuming, labour intensive, and sometimes complex laboratory procedures. Especially in the perspective of applied research, where sensors are used in real settings, the key performance indicators include the robustness and easiness of application of the detection method. It is then required to provide a sample appropriate for the sensor, which often requires a laboratory preparation not compatible with most of industrial applications.

In the case of milk, the presence of fat, proteins, and many other components in a complex phase equilibrium poses particular challenges for analysis. The two major elements in milk microstructure are casein micelles and fat globules. Casein micelles are a mixture of caseins $\left(\alpha 1-, \alpha 2^{-}, \beta-\right.$, and kcasein), which is the most abundant protein family in milk (78\%), with associated calcium phosphate. The structure of the micelle can be modelled as a cluster of submicelles linked together by calcium bridges or alternatively flexible array of casein molecules interlinking calcium phosphate nanoclusters. In both models, the micelle is stabilized by a surface covering of $\mathrm{k}$-casein providing both steric and electrostatic repulsion. Casein micelles have colloidal dimensions (50$250 \mathrm{~nm}$ ). Caseins are known as blocking agent for surfaces and therefore may interfere with sensor surfaces. 
Fat globules are composed of triglycerides with minor contributions from diacylglycerols, monoacylglycerols, free (unesterified) fatty acids, phospholipids, and sterols and are bounded by a membrane. Trace amounts of fat-soluble vitamins, carotene, and fat-soluble flavouring compounds are also present. The native fat globule membrane is complex, consisting of an inner phospholipid unit membrane $0.1 \mathrm{~nm}$ thick with associated lipoproteins, glycoproteins, enzymes, and carotene. Although protein accounts for $2 \%$ of the weight of the fat globule, the total interfacial area of the membrane is $80 \mathrm{~m}^{2} / \mathrm{L}$ or $2 \mathrm{~m}^{2} / \mathrm{g}$ fat and can have a significant influence during milk processing. The fat globule size is between 0.1 and $10 \mu \mathrm{m}$ in diameter (the range and mean vary with the species, breed and health of the animal, stage of lactation, etc.), where $90 \%$ of globules are between 1 and $8 \mu \mathrm{m}$. The remaining serum phase contains dispersed whey proteins and dissolved lactose as major components [1-3]. Fat can accumulate on surfaces or even clog narrow channels in particular conditions.

The water-based fraction includes several components, where sugars and water-soluble proteins (or whey proteins) are the primary part. Lactose is the principal carbohydrate in the milk of most species (0-10\%) and it is unique to milk [4]. Serum or whey proteins include $\alpha$-lactalbumin, $\beta$-lactoglobulin, blood serum albumin (BSA), and immunoglobulins (Ig) and account for $17 \%$ of proteins. Milk proteins also include milk fat globule membrane proteins and a large variety of enzymes (about 60) and hormones [5].

In addition to its complexity, the phase structure and composition can be influenced by processing. For instance, the temperature influences the status of immunoglobulins, which are linked to the behaviour of fat and casein membranes. Physical processing is also used to modify the milk microstructure; for example, impact of a high pressure milk stream on a surface results in the homogenisation of the fat globule size during industrial processing. This process prevents cream separation in milk usually available in the market.

In this paper, we analyse the state of the art of sample preparation for milk by comparing traditional industrial procedure with potential for miniaturisation and emerging microfluidic methods, with a particular emphasis on contaminant detection and especially for aflatoxin $M_{1}$ as case study. Most of techniques discussed in the paper, however, are related to phase and chemical separation and can be applied to other applications.

The aflatoxin $M_{1}$ is of particular interest because it is a milk contaminant and potent carcinogen classified in group 1 of the International Agency for the Research on Cancer [6]. It is characterised by carcinogenic, genotoxic, teratogenic, hepatotoxic, immunosuppressive, and antinutritional effects [7, 8]. Aflatoxin $M_{1}$ (Figure 1) is a metabolite of aflatoxin $B_{1}$. Aflatoxin $B_{1}$ is produced by the ubiquitous fungus Aspergillus flavus. In favourable climatic condition, the fungus produces aflatoxin $B_{1}$ and contaminates animal feed. Following the ingestion of spoiled feed, aflatoxin $B_{1}$ is hydroxylated creating in the liver aflatoxin $M_{1}$. Aflatoxin $M_{1}$ is secreted into milk, with an elapsed time of about 12 hours and a peak time of

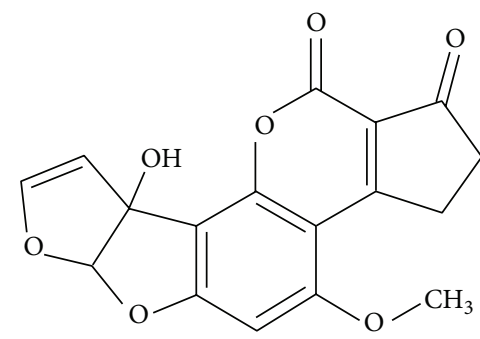

FIgURE 1: Chemical structure of aflatoxin $M_{1}$.

about 24 hours. It is thermostable and thus is not deactivated by pasteurisation or UHT treatment [9] and it is found in dairy products and concentrated in cheese [10].

In order to protect public health and safety, in the European Commission (EC) regulation number 1881/2006 the maximum level of aflatoxin $M_{1}$ contamination in milk is set to $50 \mathrm{ppt}$ and to $25 \mathrm{ppt}$ for infant formulae, as a vulnerable group of the population. Similar regulatory limits are implemented in other countries in Africa, Asia, and Latin America, where aflatoxins incidence is higher than in the EU countries due to favourable warm and wet climatic conditions. In other countries, such as the United States and some countries in Asia and Latin America, the suggested limit is only $500 \mathrm{ppt}[11,12]$.

Aflatoxin is slightly soluble in water $(10-30 \mu \mathrm{g} / \mathrm{mL})$ but yet much higher than maximum allowed limit in milk; it is insoluble in nonpolar solvents, which results in a very small fraction-about 5 to $20 \%$ depending on the source and conditions-partitioned in the cream part of milk [10]. In milk it is mainly weakly absorbed onto milk proteins [13], mainly casein. The small molecule size of aflatoxin (MW: $328.3 \mathrm{~g} / \mathrm{mol}$ ) is an additional challenge if detection is based on label-free methods monitoring the change of surface properties like measurement of refractive index (photonic sensors) or impedance (electrochemical capacitive sensors or impedance spectroscopy) at the surface.

With this background, it is clear that phase and chemical separation are needed for an efficient detection of aflatoxin. Many microfluidic methods are available for this purpose [14], but they are usually developed for small samples (often blood). Since contaminants like aflatoxin are found at very low concentration, it is often required to work with sample volumes in the range $1-100 \mathrm{~mL}$ to have a sufficient recovery for the analysis and to get a representative and uniform sample from the batch. Scaling up processing rate of microfluidic methods to deal with 1-100 mL samples in minutes is not practical in most cases and therefore a careful selection of the technology must be performed by including performances of separation and processing time.

The paper is organised as follows: in Section 2, we describe the techniques used for phase separation and sample preparation in the specific case of milk and aflatoxin. We compare the techniques used in laboratories to prepare the samples for analysis with state-of-the-art methods like ELISA and HPLC and lateral flow to industrial methods for phase separation and microfluidic techniques. A discussion of 
advantages and applications of different methods is reported in Section 3, where we discuss the role and perspective of microfluidic methods.

\section{Separation and Sample Preparation Techniques}

2.1. Laboratory Techniques. Laboratory analysis of sample can be performed with different aims and with a range of techniques. Analytical techniques used are HPLC, which is the standard confirmatory method in regulations, fluorimetry, and ELISA or lateral flow kits for screening. In certified laboratories, analyses are usually performed with reference methods to provide the best accuracy and recovery achievable and with the goal to confirm the results of screening on field. Due to the complex phase structure of milk and purpose of such procedures, extraction methods are used to reduce matrix interference and to provide the best toxin recovery from the sample. Time and complexity are usually not an issue for such methods, since the number of samples processed is lower than in screening tests. The fat fraction is usually discarded before measurement of aflatoxin content, to avoid performance reduction due to clogging of structures, interference or inactivation of immunoaffinity receptors, and so forth. Other milk components may interact with aflatoxin by reducing the recovery of toxin from sample and interfere with detection; sample preparation is often a key procedure for good analytical accuracy in the detection of AFM1. In this paragraph, we describe a summary of the most common preparation step.

\subsubsection{Centrifugation, Ultracentrifugation, and Ultrafiltration} Phase Separation. Centrifugation can be used to fractionate milk into individual phases for further analysis or processing. Defatting milk by centrifugation is a common procedure (typical procedure: $2000 \times \mathrm{g}, 10^{\prime}$ or $4000 \times \mathrm{g} 15^{\prime}$ ), but it is also possible to use an ultracentrifuge to remove casein from milk due to the nanometric particle structure, where a typical procedure is $80,000 \times \mathrm{g}$ at $4^{\circ} \mathrm{C}$ for $45 \mathrm{~min}$. In addition, the use of a cut-off membrane (e.g., 10,000 Da) can further remove milk components above a threshold weight, which is typically used to remove proteins. Procedures are batch procedures and usually time-consuming [15].

2.1.2. Clean-Up Columns. Solid phase extraction, coated liquid/liquid phase separation, and immunoaffinity are alternative techniques for sample preparation and suspension. They are traditionally used for extraction of trace components from large volumes in combination with analytical techniques. Preparation columns for aflatoxin are available on the market using immunoaffinity (aptamers, molecularly imprinted polymers, or antibodies). Similarly, solid phase extraction uses fibres or adsorbent phase made of appropriate materials to trap specific molecules, with a similar approach to chromatography capillary columns with appropriate stationary phase ranging from all-purpose $\mathrm{Cl} 8$ coating to specific coatings. Laboratory instrumentations able to perform such preparation are commonly available on the market $[16$, 17].
One key point for clean-up columns, especially when used in combination with biosensors, is the definition of release buffer, which should not be an organic solvent as conventionally used in clean-up columns for HPLC and should not interfere with the immunoaffinity sensor. Release from immunoaffinity columns can be performed by $\mathrm{pH}$ shock and reequilibration or temperature in a specific suspension buffer.

2.1.3. Solvent Extraction. Solvent extraction is the traditional technique for aflatoxin separation and concentration in HPLC analysis procedure [18]. Extraction is traditionally performed in chloroform or other organic solvents, which are purified with columns, evaporated, and resuspended in the solvent of choice for the analysis (typically water/methanol for HPLC).

As reported in the literature, it is possible to extract the aflatoxin without the use of organic solvents in milk preparation, by adding sodium citrate to milk and applying a temperature treatment [19]. With such an approach, chelation of calcium ions by citrate or EDTA [20] results in an increase of hydration of casein micelles and proteins are resuspended into water, with an increase of aflatoxin availability for detection. Organic solvents should be avoided in extraction procedure to reduce the compatibility issues of polymeric structures and safety issues in the management of consumables and compatibility with aptamers on the sensor, which are denatured in nonwater solutions. Extraction procedures typically provide a factor 2 improvement in binding to ELISA plates as reported in [19].

More recently, in several new commercial rapid kits the extraction procedure is often avoided to speed up the detection, since the time-for-result is a key performance parameter for users. However, the kits are prone to matrix interference and must be validated for a specific matrix, still providing accuracy issues and a general overestimation in the quantification of the analyte [21,22], although this is acceptable for commercial screening tools.

Lateral flow is both a laboratory method used for screening of samples and a microfluidic technique coupling intrinsic sample preparation and detection. The approach provides inexpensive and easy-to-use analysis and it is used by many commercial devices like Charm ROSA [23] for integration of a competitive ELISA test. The use of a membrane, typically in nitrocellulose, allows for intrinsic filtering, while the use of surfactants in the coupling pad to support the flow into the nitrocellulose membrane and avoid clustering of nanodot reporters is also supposed to perform an intrinsic aflatoxin release from complex with casein (Figure 2).

The technique is becoming a diffused approach for lowaccuracy semiquantitative testing including sample preparation in a user-friendly format. The simplified sample preparation is compatible with a semiquantitative evaluation of sample contaminations above the required limit by regulation for screening purposes, where degradation of performances or repeatability of the test is a minor issue as long as samples above the threshold are identified. The typical approach for screening methods is to minimise the occurrence of false negatives, which results in an overestimation of results and a potentially large number of false positives. The volume of 


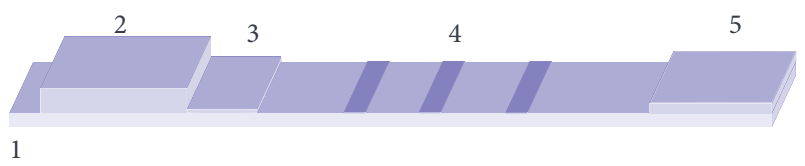

Figure 2: Lateral flow format: 1: membrane and support layer, 2: sample pad, 3: coupling pad, 4: reporting areas, and 5: adsorption pad-elaborated from [23].

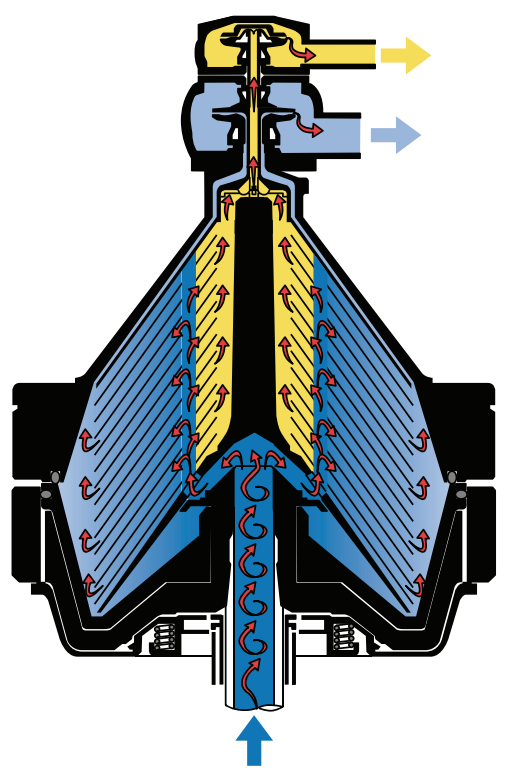

Figure 3: Centrifugal separation by rotative particle separator. Source: [24].

sample that is possible to process with such method is very limited, which may result in nonrepresentative samples in some food applications.

\subsection{Industrial Standard Techniques for Phase Separation}

2.2.1. Rotative Particle Separation. Rotative particle separation is a well-established separation technique used to skim milk in process lines with very large throughput. It is a separation technique based on density/inertia and improves the hydrocyclone separation technique by the use of a rotor to improve the efficiency and speed of separation. Milk enters in the separation bowl from the bottom and is distributed in the rotating separation plates through distribution holes. The fat fraction is concentrated at the centre of the centrifuge and collected at top of the inverted bowl (Figure 3).

The remaining fat content in the skimmed milk is usually between 0.04 and $0.07 \%$, corresponding to $1-2 \%$ of the fullmilk fat content (about 3.5\% fat) [24].

2.2.2. Cross-Flow Filtering and Ultrafiltration. Cross-flow filtering and ultrafiltration are a well-established procedure for separation of milk fractions. It is often adopted in dairies for mild pasteurisation by removing bacteria by filtering with appropriate filter size (Figure 4) [25]. A wide range of separation techniques, ranging from microfiltration to reverse osmosis, allows the separation of each fraction of milk. In particular, microfiltration removes fat and bacteria, ultrafiltration removes casein micelles, ultrafiltration/nanofiltration can further remove proteins from whey, and reverse osmosis can separate salts and inorganic compounds leaving only water in the final permeate.

Transverse-flow microfilters are designed to work with production volumes and are typically in the form of tubular ceramic, polymeric, or stainless steel filters. A longitudinal flow is maintained in the multiple bores or spiral wounded membrane of the filter, in order to provide a cross-flow with respect to separation membrane and therefore remove any deposit at filter surface [26].

A further improvement of performances is related to the possibility of using the high-speed cross-flow to "pinch" the flow through lateral pores, similar to what is used in continuous flow separation [14]. The concept is that the large particles cannot enter the thin boundary layer at channel wall that is separated through the lateral pores, because of size exclusion. The lateral flow is then free of particles above a threshold size, which can be considerably lower than actual pore size thus increasing the filter efficiency. Further effect of cross-flow is the exploitation of microfluidic forces, as described in Section 3 for inertial separation, where the larger particles tend to move to the centre of flow because of interaction with channel walls, thus reducing the filter clogging by larger particles [27].

2.2.3. Air or Gas Floatation. Air or gas floatation is a method widely used in waste processing, especially for the separation of phases of emulsions, typically oil in water or suspensions. Since many food samples are emulsions, the method can be used as a solution for sample clean-up at large volumes. The standard process includes the following steps:

(1) Coagulation or flocculation for solid suspension is promoted by chemical agents, $\mathrm{pH}$ variations, and so forth, in order to speed up the procedure in a later stage.

(2) The flow is pressurised with air or $\mathrm{N}_{2}$ flow in order to obtain a supersaturated solution of gas in the liquid phase. Gas content follows Henry's law: $P=C X$, where $P$ is partial pressure of the gas, $C$ is solubility coefficient, and $X$ is dissolved gas in the liquid phase.

(3) The liquid phase is transferred to inlet into a vessel at room pressure, thus producing a fine dispersion of gas bubbles in the bulk. Bubbles nucleate at the interface of heterogeneities of the emulsion and float to the surface, carrying the oil droplets or particles with them.

(4) Then, there is mechanical or fluidic separation of top layer, with specific structures engineered to increase the throughput. 


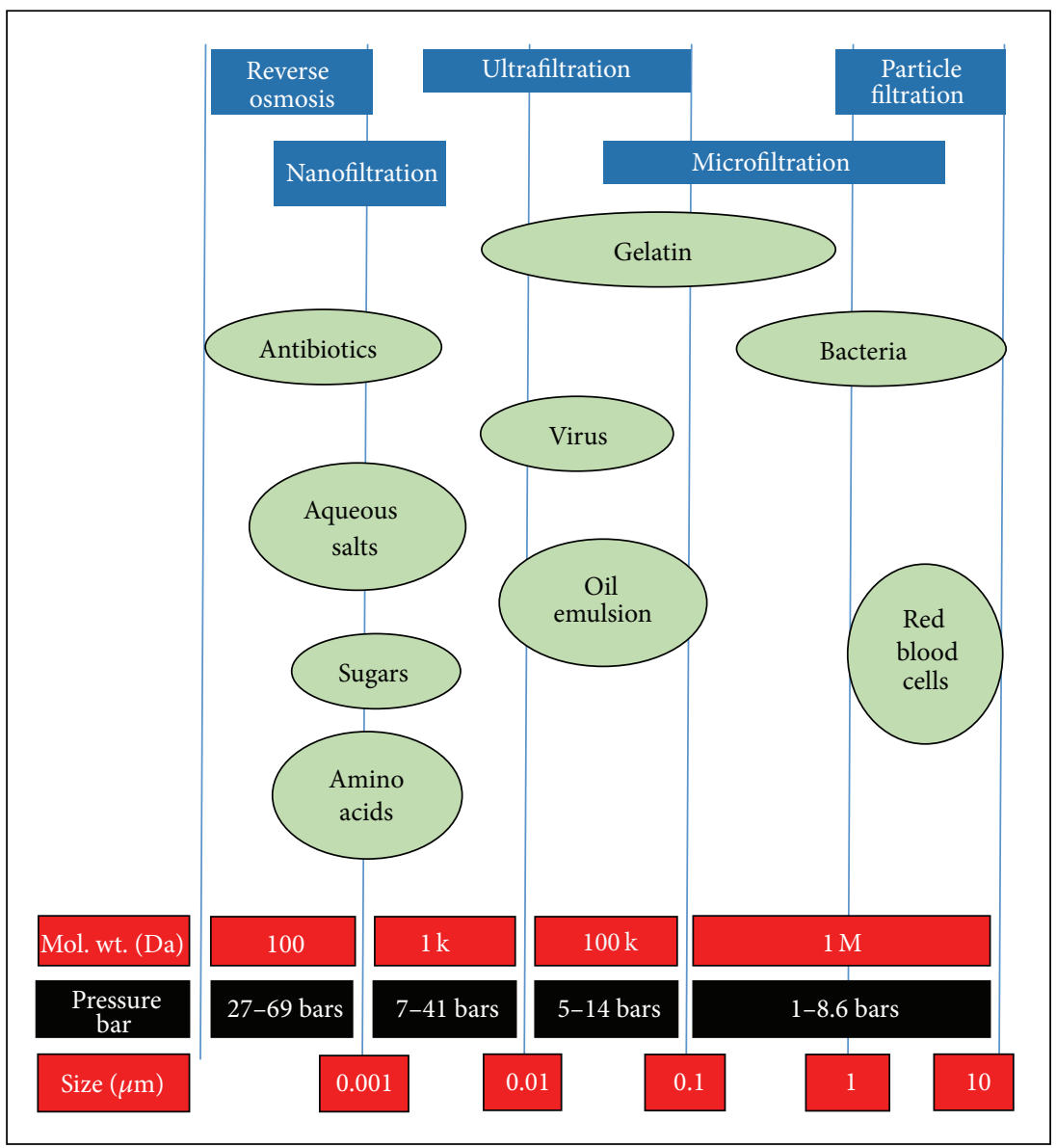

FIGURE 4: Separation ranges by different filtration techniques-elaborated from the following source: [26].

Separation follows the sedimentation law, where the sedimentation speed is

$$
v_{s}=\frac{d^{2}\left(\rho_{p}-\rho_{l}\right)}{18 \mu},
$$

where $\rho$ is the density of particle and liquid, $d$ is the particle diameter, and $\mu$ is the viscosity. When air floatation is used, a fixed ratio of dissolved gas versus particle, typically $0.0065-$ $0.08 \mathrm{mg}$ air $/ \mathrm{mg}$ particles, is used to separate particles efficiently. The floatation rate is calculated from sedimentation law, where density of the particle is corrected for air bubbles attached to the particle, thus typically reducing fat density from $0.8 \mathrm{~g} \mathrm{~cm}^{-3}$ to equivalent density of $0.015 \mathrm{~g} \mathrm{~cm}^{-3}$ (assuming uniform distribution of gas on particles). With such model, a floating velocity of about $90 \mu \mathrm{m} / \mathrm{s}$ was estimated for $10 \mu \mathrm{m}$ fat droplets. In order to achieve a real separation, such separation speed must be larger than average speed of Brownian mote $v_{B}=(k T / m)^{0.5}$. This is typically found for particles larger than $10 \mu \mathrm{m}$, which does not make this approach convenient for the purpose of milk separation without coagulation of particles before processing. A similar technique is the foam fractionation, which is reported for protein enrichment [28], based on surface affinity of bubbles toward the component to be separated, where bubbles are produced by flowing gas through a glass frit in the sample vessel.

\section{Discussion of the Role of Microfluidic Methods}

In principle, the peculiarity of microfluidic devices can be exploited to miniaturise standard separation methods or new concepts. In food applications, not all traditional advantages of microfluidics are necessarily an advantage. For instance, sample volume is often irrelevant or, even worse, the sample must be large enough to be representative of a large, nonuniform batch to be analysed. One of the major advantages of microfluidics is very precise control of the interfaces between laminar flows or phases [29] and high surface-to-volume ratio. This can be used to miniaturise the solvent extraction by putting in contact different parallel laminar flows transferring aflatoxin from a sample to a solvent stream, thus providing higher diffusion rates, leading to faster extraction procedure.

The tight control of temperature, flow rates, and volumes in microfluidic devices can provide the integration of multiple functionalities into the device, like fast thermal processing or cycling, due to small thermal mass, faster diffusion to surfaces 
for molecule trapping, or detection due to high surface-tovolume ratio. Clean-up columns can be also included as appropriate fluidic chambers with active coating, similarly to miniaturise integrated chromatography chips [30]. Thermal release from clean-up column can be largely sped up by the small volumes used, which is used, for instance, for fast thermal cycling of PCR procedures on chip. Working with large active areas over volume ratios and fine control of flow rate and temperature allows achieving concentration of analytes, which are not possible with standard clean-up concentration columns, due to large volumes of standard devices.

The new concepts of continuous flow inertial separation and electrophoretic separation are possibilities, which are made possible by a scale reduction of flow, thus increasing the speed and effectiveness of separation with approaches, which are not applicable at macroscopic scale. On the other hand, microscale fluidic forces and microfluidic concepts are already exploited to improve the performances of macroscopic systems like the role of inertial forces in the optimisation of performances of cross-flow filters, working on the scale of litres/minute in industrial plants.

In addition to peculiar functionalities, microfluidic systems can automatize bioassays and immunoaffinity procedures, for instance, by dispersing beads with appropriate coating and functionalities (e.g., magnetic beads) into a sample and recovering them by magnetic forces. Full-assay integration by mixing beads, agitation of sample, bead capture, and target analyte release and detection was demonstrated $[31,32]$. The direct integration of detection is possible, thus exploiting the small channel volumes, reduced dead volumes, and fast diffusion to surface [33]. Further down to real applications, the possibility of having high parallelisation of procedures into multiplexed devices can lead to high throughput by parallel processing of several samples at the same time.

3.1. Microfluidic Approaches to Milk Analysis. Different microfluidic approaches can be used to separate mixture components and particles, especially based on particle size, by exploiting forces generated by the interaction of particles with flow conditions and microfluidic structures. In the specific case of fat separation from milk, the required performances are

(1) to be able to separate fat particles, that is, in the size range $1-10 \mu \mathrm{m}$,

(2) to have an output throughput compatible with user requirements, that is, processing $1-10 \mathrm{~mL}$ in minutes,

(3) to be operated with simple equipment, to be selfcleaning or compatible with long term use, or to be easily replaced and cheap if disposable,

(4) low susceptibility to clogging and performance degradation.

Not all microfluidic approaches are suitable to fit with these constraints; here we discuss a selection of best candidate options available and their foreseen performances. When further separation is considered, for instance, chemical or casein separation, a combination of different techniques can be integrated in a single device to reach the required performances. In this section, we discuss the most promising approaches for sample preparation in milk.

3.1.1. Inertial Microfluidics. Inertial microfluidics is a branch of microfluidics dealing with flow condition in which a laminar flow in a microchannel is coupled with intermediate (1-100) Reynold's numbers, thus providing a limited, but still effective, inertial force on flow stream, thus exploiting peculiar effects and opportunities for separation of emulsions or suspensions. At that scale, there is a complex interaction of particles with flow and channel walls resulting in a variety of behaviours depending on channel geometry (size, aspect ratio, and eventual bending), particle properties (size, particle size-to-channel ratio, and particle concentration), and flow conditions (Reynold's number, shear rate, and Peclet's number). The position and conditions for focusing are related to two major forces, that is, shear lift and wall repulsion [34].

Shear lift can be expressed as

$$
F_{L}=\frac{4 \rho C_{L} U_{f}^{2} a^{4}}{3 \pi \mu D_{h}^{2}},
$$

where $\rho$ and $\mu$ are density and viscosity of fluid, a particle dimension, $D_{h}$ is hydraulic diameter of channel, $U_{f}$ is flow velocity, and $C_{L}$ is lift coefficient. Since $C_{L}$ is proportional to $W^{2} /\left(a^{2} \operatorname{Re}^{0.5}\right)$, with $W$ channel dimension, the overall shear force moving particles toward walls is inversely proportional to $a^{2}$. The wall repulsion depends on a similar equation, but $C_{L}$ is proportional to $H^{2} /\left(a \mathrm{Re}^{0.5}\right)$, where $H$ is the minimum channel dimension, and it is proportional to $a^{3} / \delta$, where $\delta$ is the distance from channel wall. Larger particles are then concentrated to a position toward the centre of flow stream with respect to smaller particles.

Length required for focusing is

$$
L=\frac{3 \pi \mu D_{h}^{2} L_{m}}{2 \rho C_{L} U_{f} a^{3}},
$$

where $L_{m}$ is the travelling distance. Optimal condition for particle focusing, that is, minimisation of focusing length, is usually achieved for $\mathrm{Re}$ in the range 20 to 100 depending on particle size (from experimental results, Figure 3 in [34]). Typical focusing length for particles with diameter around $10 \mu \mathrm{m}$ is in the order of millimetres for shear focusing toward channel walls and about 1-2 centimetres for shear focusing toward channel centre. Particles then focus to equilibrium positions at the centre of channel walls, especially at the centre of wider channel sides for high aspect ratio sections.

The flow regime where separation can be achieved follows the assumptions [35]:

$$
\lambda=\frac{a}{D_{h}}>0.07
$$

Then, maximum hydraulic diameter is fixed for a given particle size to be separated; as a reference, for particles of $1 \mu \mathrm{m} D_{h}$ must be less than $14 \mu \mathrm{m}$ and for $4 \mu \mathrm{m} D_{h}<56 \mu \mathrm{m}$. The other condition for particle Reynold's number $\operatorname{Re}_{p}$ is

$$
\operatorname{Re}_{p}=\operatorname{Re} \lambda^{2}>1,
$$


thus setting $\operatorname{Re}>204$, unless $\lambda$ is large (i.e., small hydraulic diameter versus particle size, where minimum Re can be, e.g., as low as 20).

When channels are bended, effect of inertial forces in flow regimes at intermediate $\mathrm{Re}$ can result in a lateral asymmetric focusing due to inertial effect [36], namely, Dean forces related to Dean number De $=\operatorname{Re} \delta^{1 / 2}$, where the curvature ratio $\delta=D_{h} / 2 r$ compares the hydraulic diameter and curvature radius $r$. According to experimental results in [35], optimal condition for lateral migration is $\lambda / \delta^{1 / 2}=2$, that is, $\delta>0.001225$, or $2 r$ less than $1.1-4.5 \mathrm{~cm}$ with $D_{h}$ of $14-60 \mu \mathrm{m}$, respectively.

A combination of inertial and hydrodynamic forces can be therefore used for the separation of fat particles at high flow rate since they are in the 1 to $10 \mu \mathrm{m}$ size range while there is no expected separation of casein, which is much smaller and it is not effectively separated with this technique.

As an additional opportunity, acoustic separation in continuous flow devices can provide a further separation method, by exploiting the fat acoustic contrast factor versus proteins and matrix. Transversal separation as in [37] may be tailored to perform high efficiency separation of fat and proteins from milk. Separation of particles with such techniques was demonstrated for beads and proteins. The separation force is

$$
F_{R}=-\left(\frac{P_{0} V_{p} \beta_{m} \pi}{2 \lambda}\right) \phi(\beta, \rho) \sin \left(\frac{4 \pi x}{\lambda}\right),
$$

where the contrast factor $\varnothing$ depends on density $\rho$ and compressibility $\beta, P_{0}$ is pressure amplitude, $V_{p}$ is particle volume, and $\lambda$ is wavelength. Although casein enrichment was demonstrated in the cited paper, the flow rate was quite low (not reported in the paper for best performing case but inferred from the text as $10-30 \mu \mathrm{L} / \mathrm{min})$. By applying this model, it was not possible to demonstrate the scalability of the design to achieve separation of particles down to $1 \mu \mathrm{m}$ size at flow rates in the $\mathrm{mL} / \mathrm{min}$ range as required by the application.

3.1.2. Electrophoresis. Electrophoresis is a common technique used for the analysis of mixtures, where the different mobility of molecules under an electric field is used to separate the components and analyse them with an appropriate detector. In such configuration, the volume of sample is quite small and separation rate is low. Electrophoresis can be also used to purify or separate species for production or sample preparation with a different configuration of the device: in the case of sample preparation, the use of electrophoresis in continuous flow devices can select charged molecules and particles in the sample and move them into a specific stream of flow that can be separated downstream. Electrophoretic separation in milk can provide a casein-enriched or depleted phase, by exploiting the charged nature of proteins at milk $\mathrm{pH}$. Electrophoresis can be used to separate effectively molecules and it is expected to scale better than body forces like inertial separation for small particles. While inertial force scales with $L^{3}$, surface charge scales with $L^{2}$ and drag resistance with $L$; therefore electrophoresis scales better than bulk forces like acoustic or inertial separation, for instance, and
TABLE 1: Example of mobility of ions, sugars, and proteins in milk.

\begin{tabular}{lcc}
\hline & Mobility & Unit \\
\hline Casein & $8.5010^{-11}$ & $\mathrm{~m}^{2} / \mathrm{s}$ \\
Lactose & $4.9010^{-10}$ & $\mathrm{~m}^{2} / \mathrm{s}$ \\
$\mathrm{Cl}^{-}$ & $2.0310^{-9}$ & $\mathrm{~m}^{2} / \mathrm{s}$ \\
\hline
\end{tabular}

the efficiency of separation for small particles performs better. Transversal separation as in [38] may be tailored to perform the separation of protein from milk. The system was already demonstrated for protein separation in the literature, although challenges related to milk composition and toxin partition into milk phases need to be investigated and a specific protocol must be set up.

The electrophoretic mobility as function of ionic strength can be modelled by the Onsager and Fuoss model [39], and in particular mobility is reduced with (ionic concentration) $)^{0.5}$, and, at the ionic strength of milk, $I=(1 / 2) \sum c_{1} z_{i}^{2}=0.08 \mathrm{M}$ [40], the expected reduction of mobility of species can be up to about $50 \%$, as reported in [39, Figure 1].

Drag velocity is calculated as $v=\mu E$, where the mobility $\mu$ of a charged chemical species in a solution is approximated as

$$
\mu_{i}=\frac{\left|z_{i}\right| e}{\left(6 \pi \eta r_{i}\right)},
$$

where $z$ is the ion charge, $e$ is the electronic charge, $\eta$ is the solvent viscosity, and $r$ is the ion radius [41]. By interacting with the solution, an electrical double layer is formed, thus altering the charge distribution in the molecule or particle surroundings. For proteins, in particular, it is common practice to evaluate the electrical mobility as a consequence of double layer formation around the molecule, thus resulting in a drag by electric field proportional to Zeta potential of the particle:

$$
\mu_{i}=\frac{\zeta \varepsilon}{\eta} .
$$

The Zeta potential of the chemical compound depends on $\mathrm{pH}$ of the solution, where the separation is performed. For proteins in particular the isoelectric point, that is, the $\mathrm{pH}$ where the molecule has neutral charge in solution, is found as tabulated data. Typical mobility of different milk components is reported in Table 1.

The use of transverse separation allows efficient separation with electrophoretic displacements in the order of 10 to 100 microns, which should allow for high performance separation with sufficient throughput.

\section{Conclusions}

In conclusion, microfluidics can play a significant role in analytical system by providing methods to increase sensitivity, accuracy, and reproducibility of detection in real samples. The small size of microfluidic structures and peculiar phenomena at microscale can be exploited to improve the speed of processing with respect to conventional laboratory techniques, while the increased system integration can reduce the work 
burden to perform laboratory test on food matrices. This approach follows the research trend of increasing blending of biochemistry, physics, engineering, and food science to produce new micronanobiosystems able to take over real-life challenges related to food safety, health, and food production.

\section{Conflict of Interests}

The authors declare that there is no conflict of interests regarding the publication of this paper.

\section{Acknowledgment}

This work has received funding from the European Union's Seventh Framework Programme FP7-ICT-2013-10 under Grant Agreement no. 610580, STREP Project "SYMPHONY."

\section{References}

[1] M. Auty, "Microscopy (microstructure of milk constituents and products)," in Encyclopaedia of Dairy Sciences, J. W. Fuquay, Ed., Academic Press, 2nd edition, 2011.

[2] M. W. Taylor and A. K. H. MacGibbon, "Milk lipids-general characteristics," in Encyclopaedia of Dairy Sciences, J. W. Fuquay, Ed., Elsevier, 2nd edition, 2011.

[3] P. F. Fox, "Fat globules in milk," in Encyclopaedia of Dairy Sciences, J. W. Fuquay, Ed., 2nd edition, 2011.

[4] P. F. Fox, "Milk-introduction," in Encyclopaedia of Dairy Sciences, J. W. Fuquay, Ed., Academic Press, 2nd edition, 2011.

[5] D. Dupont, R. Grappin, S. Pochet, and D. Lefier, "Milk proteins-analytical methods," in Encyclopaedia of Dairy Sciences, J. W. Fuquay, Ed., Academic Press, 2nd edition, 2011.

[6] International Agency for Research on Cancer (IARC), Monographs on the Evaluation of the Carcinogenic Risk of Chemicals to Humans, International Agency for Research on Cancer (IARC), Lyon, France, 1993.

[7] J. H. Williams, T. D. Phillips, P. E. Jolly, J. K. Stiles, C. M. Jolly, and D. Aggarwal, "Human aflatoxicoses in developing countries: a review of toxicology, exposure, potential heath consequences, and interventions," The American Journal of Clinical Nutrition, vol. 80, no. 5, pp. 1106-1122, 2004.

[8] P. B. Wangikar, P. Dwivedi, N. Sinha, A. K. Sharma, and A. G. Telang, "Effects of aflatoxin $B_{1}$ on embryo fetal development in rabbits," Food and Chemical Toxicology, vol. 43, no. 4, pp. 607615, 2005.

[9] M. Carvajal, A. Bolaños, F. Rojo, and I. Méndez, "Aflatoxin $\mathrm{M}_{1}$ in pasteurized and ultrapasteurized milk with different fat content in Mexico," Journal of Food Protection, vol. 66, no. 10, pp. 1885-1892, 2003.

[10] L. Anfossi, C. Baggiani, C. Giovannoli, and G. Giraudi, "Occurrence of aflatoxin M1 in dairy products," in Aflatoxins, I. TorresPacheco, Ed., InTech, Rijeka, Croatia, 2011.

[11] FAO, "Worldwide regulations for mycotoxins in food and feed in 2003," Corporate Document Repository, Food and Agriculture Organisation, 2011.

[12] US-FDA, Whole Milk, Low fat Milk, Skim Milk: Aflatoxin M1 $C P G$, section 527.400, 2005.

[13] IARC, The Monographs, vol. 100F, IARC, 2012.

[14] N. Pamme, "Continuous flow separations in microfluidic devices," Lab on a Chip, vol. 7, no. 12, pp. 1644-1659, 2007.
[15] A. Barbiroli, F. Bonomi, S. Benedetti et al., "Binding of aflatoxin $\mathrm{M}_{1}$ to different protein fractions in ovine and caprine milk," Journal of Dairy Science, vol. 90, no. 2, pp. 532-540, 2007.

[16] "Website of Thermo Scientific," 2015, https://www.thermoscientific.com/en/products/automated-solid-phase-extraction.html.

[17] Agilent, "Simultaneous determination of 26 mycotoxins in sesame butter using modified QuEChERS coupled with UHPLC-ESI/triple quadrupole mass spectrometry," Application Note 5991-5347EN, Agilent, 2015.

[18] Macrae, HPLC in Food Analysis, Academic Press, 1988.

[19] L. Anfossi, M. Calderara, C. Baggiani, C. Giovannoli, E. Arletti, and G. Giraudi, "Development and application of solvent-free extraction for the detection of aflatoxin $\mathrm{M}_{1}$ in dairy products by enzyme immunoassay," Journal of Agricultural and Food Chemistry, vol. 56, no. 6, pp. 1852-1857, 2008.

[20] M. C. A. Griffin, R. L. J. Lyster, and J. C. Price, "The disaggregation of calcium-depleted casein micelles," European Journal of Biochemistry, vol. 174, no. 2, pp. 339-343, 1988.

[21] W. Li, S. Powers, and S. Y. Dai, "Using commercial immunoassay kits for mycotoxins: 'Joys and sorrows'?" World Mycotoxin Journal, vol. 7, no. 4, pp. 417-430, 2014.

[22] A. J. Alldrick, "Looking for best compromise in rapid food mycotoxin tests: speed, sensitivity, precision and accuracy," World Mycotoxin Journal, vol. 7, no. 4, pp. 407-415, 2014.

[23] S. J. Saul, M. E. Tess, and R. J. Markovsky, "Lateral flow test kit and method for detecting an analyte," US Patent no. US 7,785,899 B2, Charm Sciences, Lawrence, Mass, USA, 2010.

[24] Tetra Pak, "Centrifugal separators and milk fat standardisation," in Dairy Processing Handbook, chapter 6.2, p. 98, Tetra Pak, 2003.

[25] G. T. Vladisavljevic, M. Shimizub, and T. Nakashima, "Preparation of monodisperse multiple emulsions at high production rates by multi-stage premix membrane emulsification," Journal of Membrane Science, vol. 244, no. 1-2, pp. 97-106, 2004.

[26] GEA filtration, August 2015, http://www.geafiltration.com/technology/cross_flow_filtration.asp.

[27] A. M. C. van Dinther, C. G. P. H. Schroën, A. Imhof, H. M. Vollebregt, and R. M. Boom, "Flow-induced particle migration in microchannels for improved microfiltration processes," Microfluidics and Nanofluidics, vol. 15, no. 4, pp. 451-465, 2013.

[28] J. Merz, H. Zorn, B. Burghoff, and G. Schembecker, "Purification of a fungal cutinase by adsorptive bubble separation: a statistical approach," Colloids and Surfaces A: Physicochemical and Engineering Aspects, vol. 382, no. 1-3, pp. 81-87, 2011.

[29] J. Atencia and D. J. Beebe, "Controlled microfluidic interfaces," Nature, vol. 437, no. 7059, pp. 648-655, 2005.

[30] L. Sainiemi, T. Nissilä, R. Kostiainen, S. Franssila, and R. A. Ketola, "A microfabricated micropillar liquid chromatographic chip monolithically integrated with an electrospray ionization tip," Lab on a Chip, vol. 12, no. 2, pp. 325-332, 2012.

[31] L. A. Sasso, I. H. Johnston, M. Zheng, R. K. Gupte, A. Ündar, and J. D. Zahn, "Automated microfluidic processing platform for multiplexed magnetic bead immunoassays," Microfluidics and Nanofluidics, vol. 13, no. 4, pp. 603-612, 2012.

[32] C. T. Lim and Y. Zhang, "Bead-based microfluidic immunoassays: the next generation," Biosensors and Bioelectronics, vol. 22, no. 7, pp. 1197-1204, 2007.

[33] A. Mortari and L. Lorenzelli, "Recent sensing technologies for pathogen detection in milk: a review," Biosensors and Bioelectronics, vol. 60, pp. 8-21, 2014. 
[34] J. Zhou and I. Papautsky, "Fundamentals of inertial focusing in microchannels," Lab on a Chip, vol. 13, no. 6, pp. 1121-1132, 2013.

[35] J. M. Martel and M. Toner, "Particle focusing in curved microfluidic channels," Nature Scientific Reports, vol. 3, article 3340, 2013.

[36] D. Di Carlo, "Inertial microfluidics," Lab on a Chip, vol. 9, no. 21, pp. 3038-3046, 2009.

[37] C. Grenvall, P. Augustsson, J. R. Folkenberg, and T. Laurell, "Harmonic microchip acoustophoresis: a route to online raw milk sample precondition in protein and lipid content quality control," Analytical Chemistry, vol. 81, no. 15, pp. 6195-6200, 2009.

[38] N. Narayanan, A. Saldanha, and B. K. Gale, "A microfabricated electrical SPLITT system," Lab on a Chip, vol. 6, no. 1, pp. 105114, 2006.

[39] S. S. Bahga, M. Bercovici, and J. G. Santiago, "Ionic strength effects on electrophoretic focusing and separations," Electrophoresis, vol. 31, no. 5, pp. 910-919, 2010.

[40] F. Gaucheron, "Milk salts, distribution and analysis," in Encyclopedia of Dairy Sciences, J. W. Fuquay, Ed., p. 908, Elsevier, 2nd edition, 2011.

[41] C. G. Zoski, Handbook of Electrochemistry, Elsevier, 2007. 


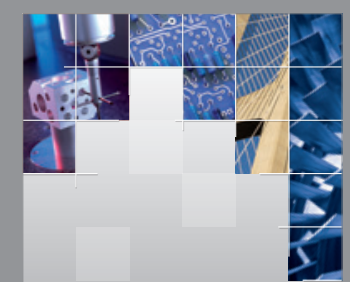

\section{Enfincering}
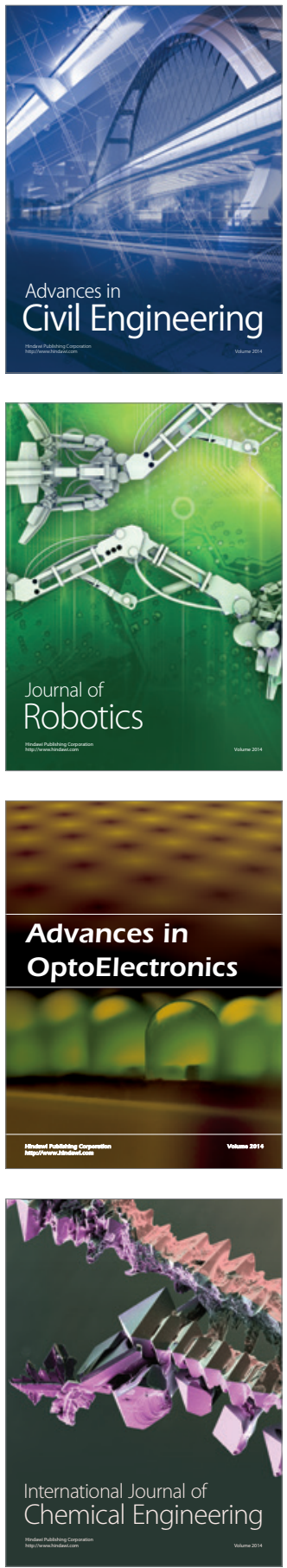

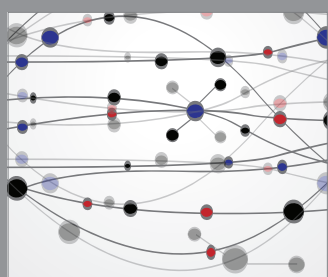

The Scientific World Journal

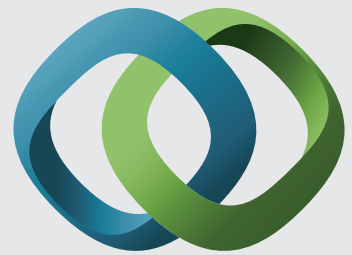

\section{Hindawi}

Submit your manuscripts at

http://www.hindawi.com
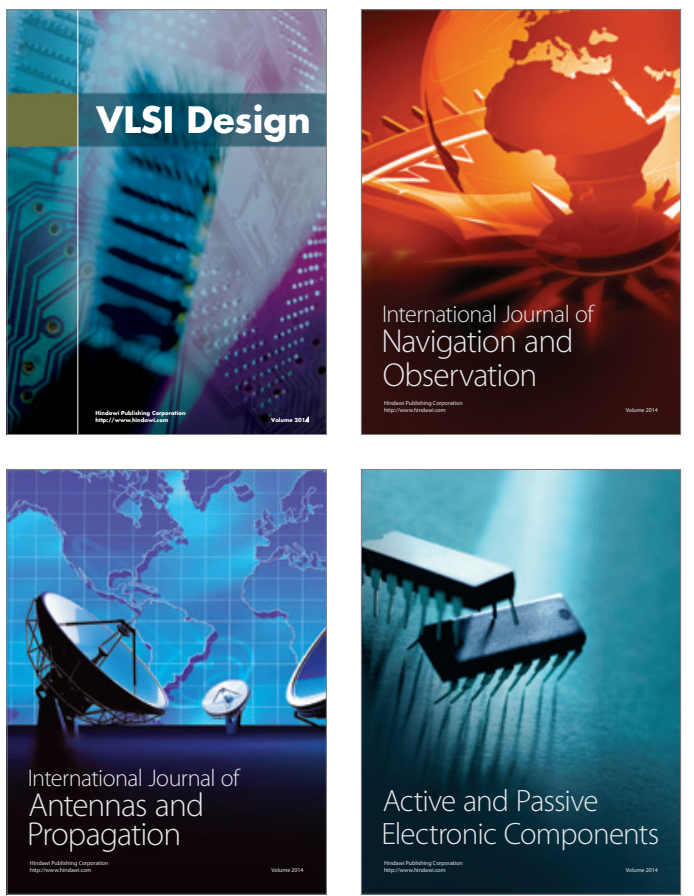
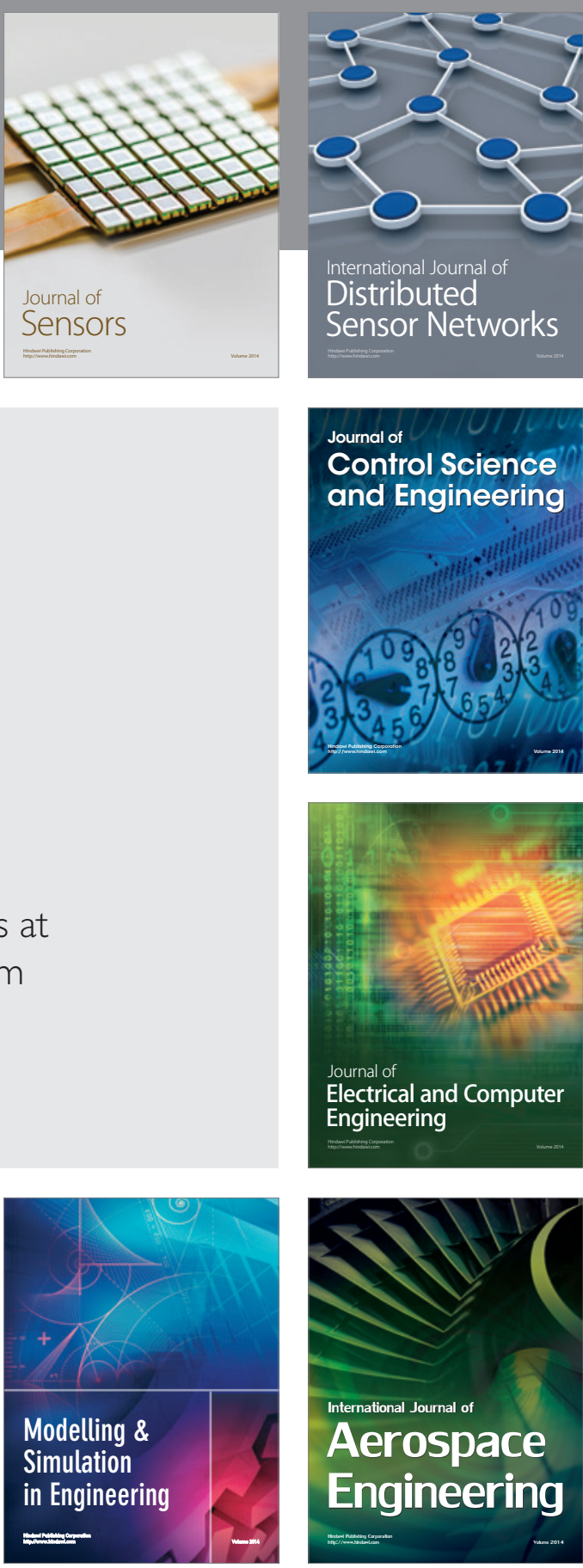

International Journal of

Distributed

Sensor Networks

Journal of

Control Science

and Engineering
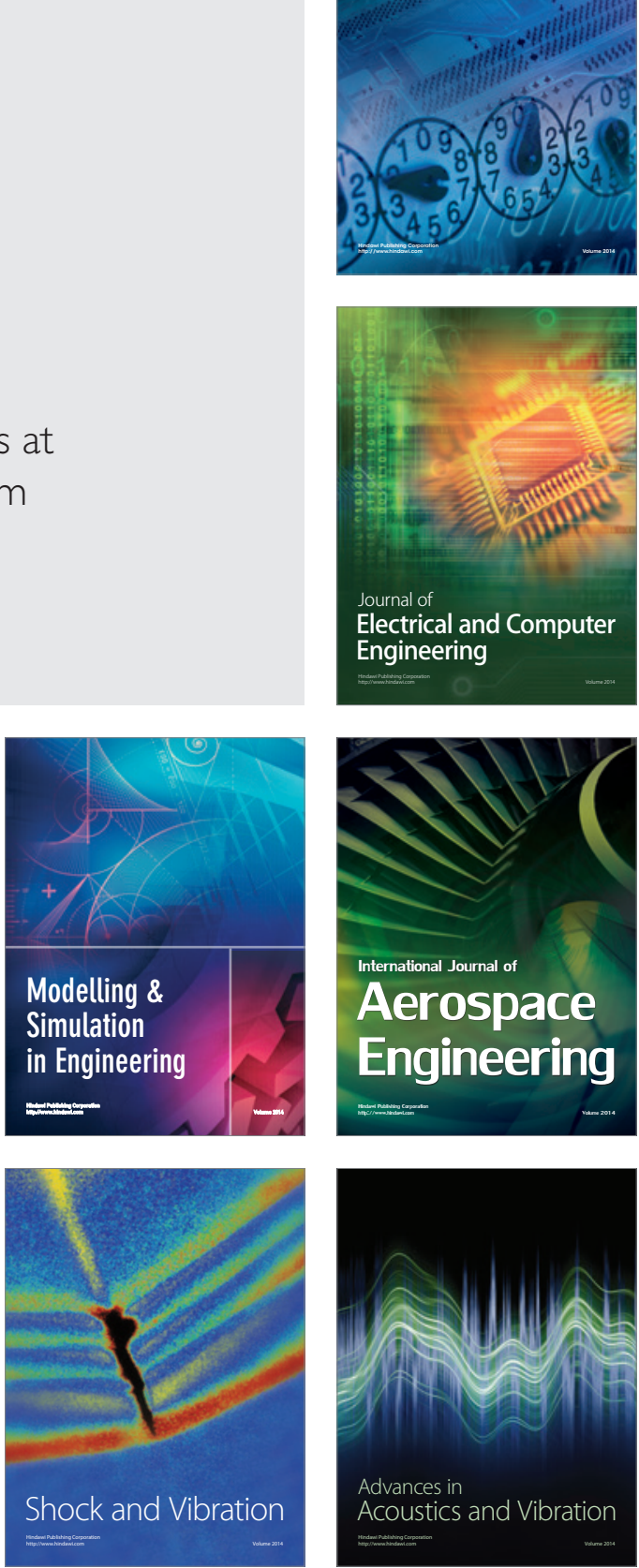